\title{
Influence of intramolecular polar bonds on interface energetics in perfluoro-pentacene on $\mathbf{A g}(111)$
}

\author{
Steffen Duhm, ${ }^{1, *}$ Shunsuke Hosoumi, ${ }^{1}$ Ingo Salzmann, ${ }^{2}$ Alexander Gerlach, ${ }^{3}$ Martin Oehzelt,,${ }^{4}$ Bernhard Wedl,, 5 \\ Tien-Lin Lee, ${ }^{6, \dagger}$ Frank Schreiber, ${ }^{3}$ Norbert Koch,${ }^{2}$ Nobuo Ueno, ${ }^{1}$ and Satoshi Kera ${ }^{1}$ \\ ${ }^{1}$ Graduate School of Advanced Integration Science, Chiba University, 1-33 Yayoi-cho, Inage-ku, Chiba 263-8522, Japan \\ ${ }^{2}$ Institut für Physik, Humboldt-Universität zu Berlin, Newtonstr. 15, D-12489 Berlin, Germany \\ ${ }^{3}$ Institut für Angewandte Physik, Universität Tübingen, Auf der Morgenstelle 10, D-72076 Tübingen, Germany \\ ${ }^{4}$ Institut für Experimentalphysik, Johannes Kepler Universität Linz, Altenbergerstr. 69, A-4040 Linz, Austria \\ ${ }^{5}$ Institut für Festkörperphysik, Technische Universität Graz, Petersgasse 12, A-8010 Graz, Austria \\ ${ }^{6}$ European Synchrotron Radiation Facility, BP 220, 38043 Grenoble Cedex 9, France
}

(Received 7 September 2009; revised manuscript received 4 November 2009; published 19 January 2010)

\begin{abstract}
We investigated the structural and electronic properties of vacuum sublimed perfluoro-pentacene (PFP) thin films on $\mathrm{Ag}(111)$ substrates using x-ray standing waves (XSW), x-ray diffraction (XRD) and ultraviolet photoelectron spectroscopy (UPS). XSW results reveal a flat adsorption geometry of the monolayer PFP/Ag(111) with a relatively large bonding distance of $3.16 \AA$ for both, the carbon and fluorine atoms. Multilayers PFP/Ag(111) adopt a herringbone structure with the molecular long axis parallel to the substrate and a vertical lattice spacing of $3.06 \AA$ as evidenced by XRD. The strong intramolecular polar bond character of the fluorine-carbon bonds in PFP leads to an orientation dependent ionization energy (IE) that is experimentally observed by UPS for the monolayer-multilayer transition: The inclined molecular plane orientation in the multilayer herringbone arrangement leads to an increase of the PFP IE by $>0.4 \mathrm{eV}$ compared to the flat lying monolayer.
\end{abstract}

DOI: 10.1103/PhysRevB.81.045418

PACS number(s): 79.60.Dp, 68.49.Uv, 72.80.Le, 68.43.Fg

\section{INTRODUCTION}

Metal-organic interface properties, such as the interfacial energy-level alignment, are key issues for the performance of devices in the field of organic electronics. ${ }^{1-3}$ However, even for weakly interacting (physisorbed) systems the complex processes determining the electronic structure at the interface are not yet fully understood. Intramolecular, intermolecular and substrate-adsorbate interactions, and their mutual interplay, are of utmost importance in this context. ${ }^{4-6}$ Recently, the impact of intramolecular polar bonds (IPBs) on the ionization energy (IE) of organic thin films was revealed ${ }^{7-10}$ and the importance of the molecular orientation relative to the substrate was highlighted. The IE of the rodlike molecule perfluoro-pentacene (PFP) (Ref. 11) [for molecular structure see Fig. 1(a)] is $0.65 \mathrm{eV}$ higher if PFP is nearly upright standing on the substrate in comparison to flat lying PFP. $^{7,10,12}$ On metallic substrates conjugated organic molecules preferentially adsorb with their $\pi$ system parallel to the surface (i.e., flat lying orientation). ${ }^{13-15}$ However, with increasing film thickness the molecules often undergo an orientational transition. ${ }^{16-19}$ In a previous ultraviolet photoelectron spectroscopy (UPS) study of PFP on $\mathrm{Au}(111)$ a transition from a flat lying monolayer to herringbonelike multilayers was proposed, ${ }^{12}$ and concomitant changes in the IE were reported, however, they could not be explained. The IPBs of PFP are expected to have a similar impact on the electronic structure of a PFP thin film irrespective whether it is composed of standing molecules or of molecules with their short axes tilted with respect to the substrate surface [Fig. 1(a)]. Thus, it may be speculated that the IE for lying molecules in the herringbone structure of PFP is larger compared to that in the flat lying conformation and, indeed, an increase of the IE was found with increasing film thickness. ${ }^{12}$ As the fluorination of pentacene (PEN) significantly reduces the adsorbate-substrate interaction, ${ }^{12,20,21}$ leading to physisorption on various metal substrates, a flat lying monolayer and tilted multilayer can be expected also on the $\operatorname{Ag}(111)$ substrate. Thus PFP/Ag(111) is a useful prototypical system to unambiguously elucidate the impact of IPBs on the electronic structure in herringbonelike arrangements.

In order to correlate the electronic structure of an organic film with an orientational transition at a certain layer thickness it is important to measure the structural properties independently. Thus, in our present study on the PFP/Ag(111) interfacial energetics and growth behavior we followed a multitechnique approach to clarify the impact of IPBs in a lying molecular conformation on the IE of the film. We performed thickness dependent measurements with the surface sensitive UPS technique in combination with x-ray standingwave (XSW) (Refs. 22 and 23) experiments, which allow determining the bonding distance of organic monolayer adsorbates on single crystalline metal substrates as well as intramolecular distortions with unsurpassed accuracy. ${ }^{24-26}$ To obtain information on the interlayer spacing and the molecular orientation in multilayers we performed specular $\mathrm{x}$-ray diffraction (XRD) investigations.

\section{EXPERIMENTAL DETAILS}

PFP (Kanto Denka Kogyo Co., Ltd.) thin films were prepared by vacuum sublimation on clean $\operatorname{Ag}(111)$ surfaces (repeated Ar-ion sputtering and annealing cycles [up to $\left.550{ }^{\circ} \mathrm{C}\right]$ ) using resistively heated quartz crucibles with deposition rates of about $0.25 \AA / \mathrm{min}$. The film mass thickness was monitored with a quartz-crystal microbalance. Hence, the values for the coverage correspond to values of nominal film thickness. XSW experiments were done in back reflec- 
(a)

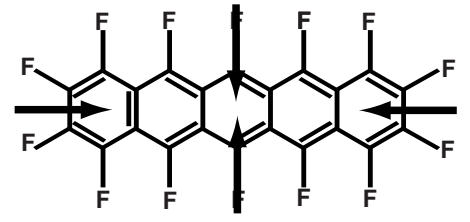

(b)

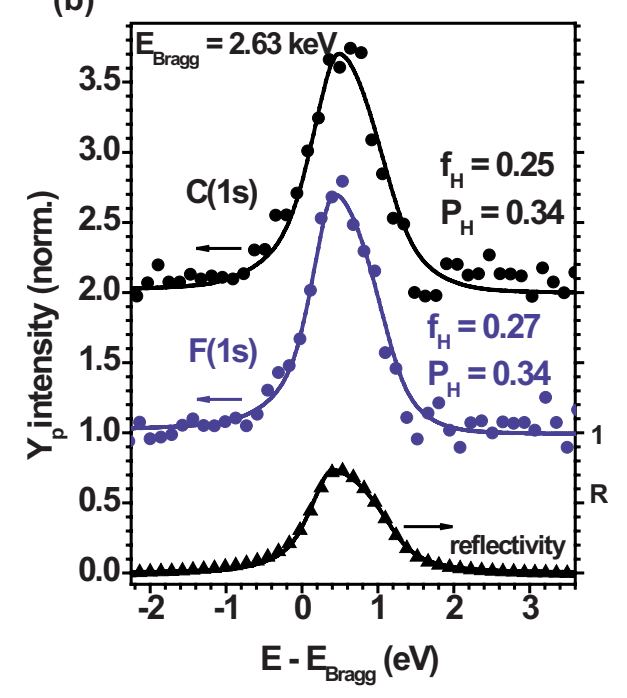

(c)

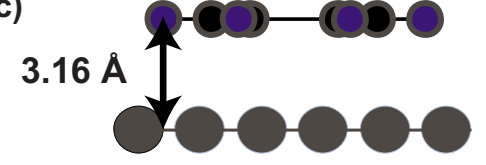

FIG. 1. (Color online) (a) Molecular structure of PFP. The arrows indicate the intramolecular dipole moments originating from IPBs. (b) The photoelectron yield $Y_{p}$ and the reflectivity $R$ as function of photon energy $(E)$ minus Bragg energy $\left(E_{\text {Bragg }}\right)$ for PFP on $\mathrm{Ag}(111)$. Symbols correspond to the experimental data and curves to the least mean-square fits according to Eq. (1). The $Y_{p}$ curves are shifted vertically to allow better visibility. For each curve the coherent fraction $\left(f_{H}\right)$ and coherent position $\left(P_{H}\right)$ are noted. (c) Sketch of a PFP molecule (carbon in black, fluorine in blue) on $\mathrm{Ag}(111)$, a view along the molecular long axis is shown. The dimensions are not to scale.

tion geometry at beamline ID32 at the ESRF (Grenoble, France $)^{27}$ with a combined preparation and analysis chamber (base pressure $3 \times 10^{-10} \mathrm{mbar}$ ). The PFP coverage being in the submonolayer regime (nominally $1 \AA$ ) was further proved by the ratio of $\mathrm{C}(1 s)$ and $\mathrm{Ag}(4 d)$ photoemission intensities. X-ray photoelectron spectroscopy (XPS) spectra for the XSW analysis were recorded with an electron analyzer (Physical Electronics) mounted at an angle of $45^{\circ}$ relative to the incoming $\mathrm{x}$-ray beam, the $\mathrm{x}$-ray reflectivity was measured using a screen inside the UHV chamber. The high photon flux necessary for XSW experiments led to a certain degradation of PFP. After $\sim 30$ min of exposure to synchrotron radiation an additional feature at the low binding energy (BE) side of the $\mathrm{F}(1 s)$ peak occurred. Thus, each XSW scan was performed at a new sample spot and the $\mathrm{F}(1 s)$ peak was checked after each scan. We never observed any additional spectral feature in $\mathrm{F}(1 s)$ spectra at lower binding energy (which would be indicative of molecule decomposition), providing confidence that $\mathrm{x}$-ray beam-induced sample damage did not compromise XSW results to a notable extent. UPS experiments were performed using a custom built apparatus equipped with a HeI UV light source and a hemispherical electron energy analyzer (Scienta R3000). The interconnected sample preparation chambers (base pressure 2 $\times 10^{-10} \mathrm{mbar}$ ) and analysis chamber (base pressure 4 $\times 10^{-10} \mathrm{mbar}$ ) allowed sample transfer without breaking ultrahigh vacuum conditions. The angle between the incident beam and the sample was fixed to $65^{\circ}$. The spectra were measured at photoelectron take-off angles $(\theta)$ of $0^{\circ}$ (normal emission) and $45^{\circ}$ (off-normal emission) with an acceptance angle of $\pm 10^{\circ}$. The energy resolution (as determined by analyzing the width of the $\mathrm{Ag}(111)$ Fermi edge) was set to 80 $\mathrm{meV}$. The error of UPS BE values is estimated to be $\pm 0.05 \mathrm{eV}$. For the measurement of the secondary electron cutoff (SECO) the sample was biased at $-3.00 \mathrm{~V}$. XRD measurements were performed at beamline W1 (Ref. 28) at HASYLAB (Hamburg, Germany) under ambient conditions using a MYTHEN 1D detector, the incident-beam energy was set to $10.5 \mathrm{keV}$. All preparation steps and measurements were performed at room temperature. Analysis of XSW data was done using the software packages xps2dare and dare (both developed at the ESRF), more details about the analysis procedure (including corrections due to nondipole contributions) can be found in Refs. 26 and 27.

\section{RESULTS}

First, the results of the XSW measurements are presented, which allow to derive the PFP (sub-)monolayer bonding distance to the $\mathrm{Ag}(111)$ substrate and possible molecular distortions. The XSW photoelectron yield $\left(Y_{p}\right)$ is given by ${ }^{22,23}$

$$
Y_{p}=1+R+2 C \sqrt{R} f_{H} \cos \left(\nu-2 \pi P_{H}\right)
$$

( $R$ : reflectivity; $C$ : polarization factor; and $\nu$ : phase factor) and allows to determine both the coherent fraction $\left(f_{H}\right)$, which is a measure for the degree of order within the adsorbate, and the coherent position $\left(P_{H}\right)$, from which the bonding distance $\left(\mathrm{d}_{h}\right)$ can be deduced. As expected, the $\operatorname{Ag}(111)$ substrate exhibited a high degree of crystallinity with $f_{H}=0.96$ and $P_{H}=0.99$. XSW of PFP/Ag(111) [Fig. 1(b)] was measured with a very low submonolayer PFP coverage of nominal $1 \AA$ to exclude contributions from PFP multilayer as PFP on $\operatorname{Ag}(111)$ exhibits pronounced island growth (see XRD and UPS results below). A PFP bonding distance to $\mathrm{Ag}(111)$ of $3.16 \pm 0.06 \AA$ was determined for both the carbon and the fluorine atoms. This evidences that PFP adsorbs (within the precision of our experiment) in an undistorted planar conformation [Fig. 1(c)] with a rather large bonding distance. $^{24-27,29}$ Furthermore, the coherent fractions of $\mathrm{C}$ $(0.25)$ and $\mathrm{F}(0.27)$ (both \pm 0.03$)$ point to an adsorption geometry with both the molecular long and short axes oriented parallel to the substrate plane. Even a relatively small tilt angle of $10^{\circ}$ of the molecular short axes would lead to a spread of the bonding distances of the PFP carbons and fluorines of $>0.5 \AA$ and thus to much lower coherent fractions.

To assess molecular orientation in multilayer films, we performed specular $\mathrm{x}$-ray diffraction on a PFP/Ag(111) film of $100 \AA$ nominal film thickness (Fig. 2). Besides a strong 


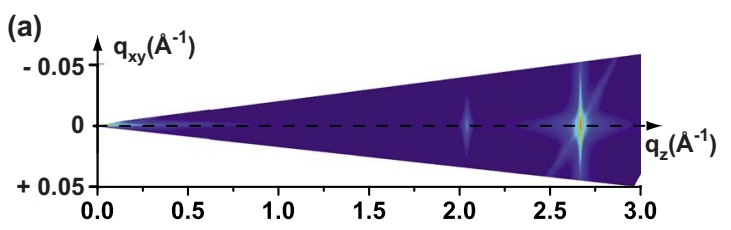

(b)

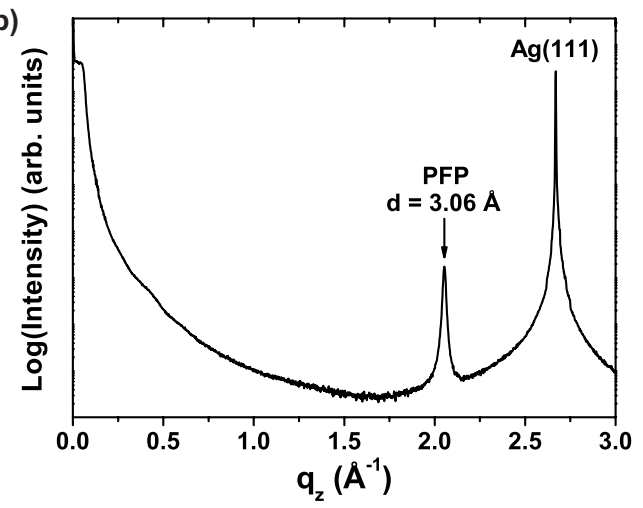

FIG. 2. (Color online) Specular XRD scan of a $100 \AA$ PFP/ $\operatorname{Ag}(111)$ film plotted as (a) intensity $(I)$ as function of the components of the scattering vector $q$ and as (b) a line scan $I\left(q_{z}\right)$ at $q_{x y}$ $=0$.

contribution of the $\operatorname{Ag}(111)$ substrate, the spectrum exhibited only one single reflection that can be attributed to the organic film, corresponding to an interlayer spacing $d$ of $3.06 \AA$. The remarkably small $q_{x y}$ width of this peak of $0.011 \AA^{-1}$ [Ag(111): $\left.0.007 \AA^{-1}\right]$ as deduced from the map in Fig. 2(a) (intensity $I$ as function of the components of the scattering vector q, i.e., $\left.I\left(q_{x y}, q_{z}\right)\right)$ by a fit along $q_{x y}$ with a pseudo-Voigt function demonstrates a high crystalline quality of the organic adsorbate. From the width of the specular PFP Bragg peak [Fig. 2(b)] the out-of-plane crystalline coherence length (estimated via the Scherrer formula) was found to exceed the nominal film thickness by more than a factor of four, thus indicating pronounced island growth of PFP/Ag(111), which is further in line with our UPS results (see below). The results prove that PFP in multilayers adopts a lying (i.e., long molecular axis parallel to the substrate surface) orientation on the $\operatorname{Ag}(111)$ substrate with no indication for the presence of standing molecules (long molecular axis vertically inclined) as commonly observed on $\mathrm{SiO}_{2} \cdot{ }^{30,31}$ No significant peaks at low values of perpendicular momentum transfer $q_{z}$ were observed, which would otherwise be indicative of (almost) standing molecules. However, the observed PFP peak in our PFP/Ag(111) spectrum cannot be indexed with any of the two known PFP crystal structures, ${ }^{11,30}$ thus indicating PFP growth in a yet unknown polymorph on $\mathrm{Ag}(111)$ substrates. From our present data a complete structure solution cannot be achieved and will be subject of a forthcoming study.

The x-ray diffraction data clearly rule out that molecules in the multilayer are standing upright. Consequently, molecules must be oriented with their long molecular axis parallel to the substrate surface. Two fundamentally different options for the orientation of the molecular plane now exist: (i) either the molecular plane is parallel to the substrate surface and "columnar stacking" of PFP occurs, or (ii) the molecular plane is inclined relative to the substrate surface, which is a herringbone-type arrangement of molecules. Option (i) can be ruled out from fundamental considerations that govern molecule crystal structures: the charge distribution in PFP is characterized by a delocalized $\pi$-electron system above and below the molecular plane and considerable negative partial charge on the $\mathrm{F}$ atoms at the periphery. The molecule has no net dipole moment but a quadrupole moment. In principle, stacking of the $\pi$-electron system in the vertical direction is a driving force toward coplanar molecules, as often observed for disklike molecules. However, the negatively charged $\mathrm{F}$ atoms repel each other via Coulomb interaction and make $\pi$ stacking impossible. Now closepacked coplanar molecules in multilayers with horizontally displaced coordinates with respect to the underlying layer might be considered. However, such an arrangement of molecules is energetically highly unfavorable because it is not found in any direction of the bulk crystal structure of PFP. ${ }^{11}$ Since multilayers are merely physisorbed on the stronger bonded PFP monolayer directly on the metal there is no thermodynamic driving force for a multilayer structure that deviates notably from the stable bulk crystal structure. Furthermore, the lattice spacing observed in our specular XRD scan of $3.06 \AA$ is significantly lower than the sum of two carbon van der Waals radii $(\sim 3.40 \AA)$ and thus lower than the expected stacking distance for PFP. ${ }^{58}$ Therefore, the observed PFP lattice spacing is too small to be explained as the distance of PFP stacking with the molecular planes parallel to the $\mathrm{Ag}(111)$ substrate plane. Consequently, a herringbone arrangement of PFP molecules in multilayers is the only rational explanation for the observed $\mathrm{x}$-ray diffraction results.

To derive the electronic structure of PFP adsorbed on $\operatorname{Ag}(111)$ we performed layer-thickness-dependent UPS measurements (Fig. 3). The deposition of up to $4 \AA$ PFP on $\operatorname{Ag}(111)$ resulted in an attenuation of $\mathrm{Ag}(111)$ spectral features (d-bands centered at about $5 \mathrm{eV}$ BE, the metal Fermi edge and the Shockley surface state) [Figs. 3(b)-3(d)]. The SECO shifted gradually to lower kinetic energy [Fig. 3(a)]; thus, the sample work function was reduced from 4.55 [pristine $\mathrm{Ag}(111)]$ to $4.13 \mathrm{eV}$. This vacuum-level shift can be mainly attributed to the electron push-back effect at the organic/metal interface, which is a common phenomenon for weakly interacting systems. ${ }^{5,32}$ Furthermore, several spectral features from the molecular adsorbate appeared, most prominent are two peaks at about 2 and $3 \mathrm{eV} \mathrm{BE}$, respectively [Fig. $3(\mathrm{~d})$, which can be assigned to the emission from the highest occupied molecular orbital (HOMO) and HOMO-1 of PFP, both of which exhibit distinctive vibronic progressions. ${ }^{12,33,34}$ The HIB (hole injection barrier; defined as energy difference between the low-BE HOMO onset and the Fermi level of the substrate) of $4 \AA$ PFP/Ag(111) was $1.67 \mathrm{eV}$ resulting in an IE of $5.80 \mathrm{eV}$. The maximum of the HOMO emission was centered at $1.82 \mathrm{eV}$ BE. Subsequent PFP deposition [6 $\AA$ PFP/Ag(111)] did not further shift the SECO position, therefore we conclude that $\sim 4 \AA \mathrm{PFP} / \mathrm{Ag}(111)$ correspond to an essentially closed PFP monolayer. The HIB was then slightly reduced to $1.56 \mathrm{eV}$, and most strikingly both the HOMO and HOMO-1 assigned spectral features appeared doubled at higher BE with the additional peaks both situated at $0.35 \mathrm{eV}$ higher BE. A similar behavior as for the $\pi$-electron derived HOMO and HOMO-1 peaks could also observed for the 


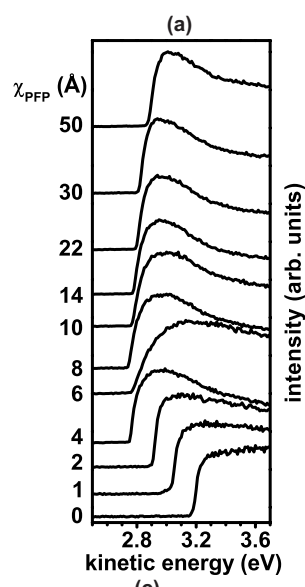

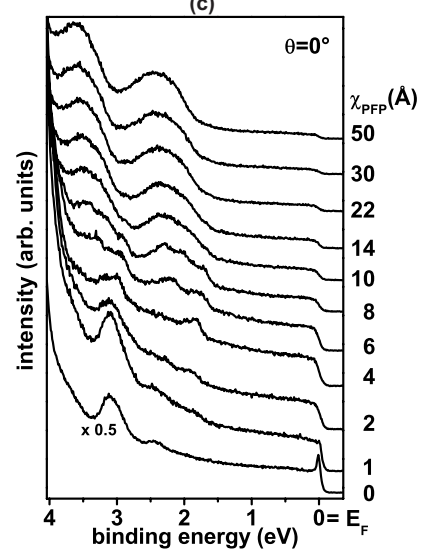

(b)

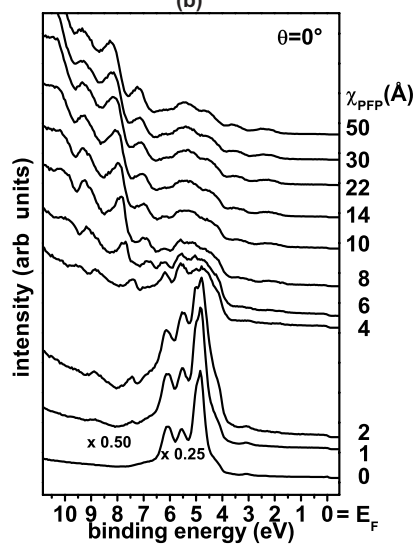

(d)

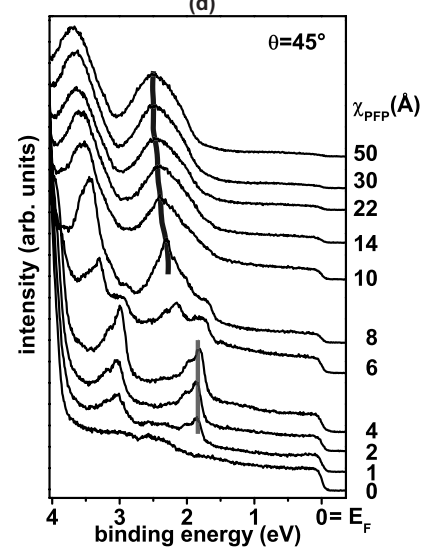

FIG. 3. UPS HeI spectra of PFP/Ag(111) showing (a) the secondary electron cutoff (SECO) region and (b) the valence electron region for an electron emission angle $(\theta)$ of $0^{\circ}$. In the bottom row the region near to the Fermi energy $\left(E_{F}\right)$ is shown on an enlarged scale for (c) $\theta=0^{\circ}$ and (d) $\theta=45^{\circ}$. In (d) the evolution of the HOMO peak maximum is highlighted by lines for the emission from monolayer PFP (light gray) and multilayer PFP in the herringbone arrangement (dark gray). $\chi_{P F P}$ denotes the nominal film thickness.

deeper lying $\sigma$-electron derived peaks, e.g., the peak centered at $7.40 \mathrm{eV}$ for monolayer coverage [Fig. 3(b)]. Thus, these emission is assigned to the second PFP layer appearing at higher binding energy compared to the monolayer due to reduced photohole screening by the organic underlayer compared to the metal substrate. ${ }^{35-37}$ The shift of the monolayer features by the deposition of the second layer is in good agreement with the UPS results of PFP/Au(111) (Ref. 12) and was explained by the increased screening of the PFP monolayer due to the additional second layer (compared to just vacuum without the second layer). For further PFP deposition the multilayer contributions beyond the second layer were found at even higher binding energy and the intensity of the monolayer and second layer emission decreased. For $8 \AA \mathrm{PFP} / \mathrm{Ag}(111)$ the multilayer HOMO was centered at $2.30 \mathrm{eV} \mathrm{BE}$ and for the final coverage of $50 \AA$ at $2.50 \mathrm{eV}$ BE. The work function increased slightly up to $4.25 \mathrm{eV}$ for $50 \AA$ PFP/Ag(111). Even for a nominal coverage of $50 \AA$ the metal Fermi edge was still visible, which confirms the island growth mode of PFP on $\mathrm{Ag}(111)$, as already found with XRD (see above). This island growth leads to a super-

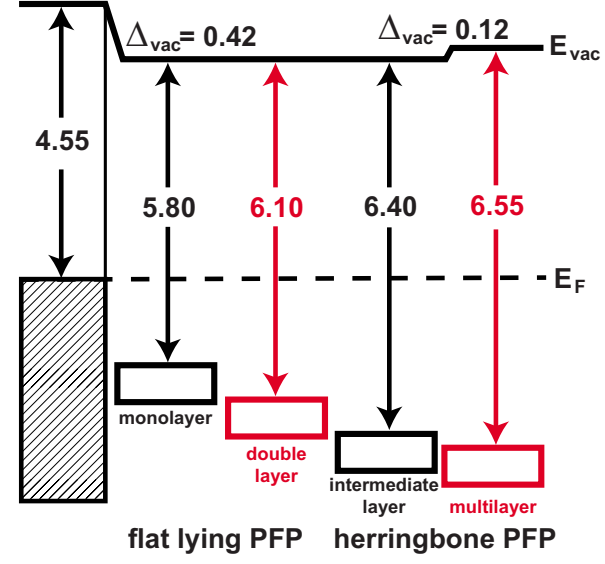

FIG. 4. (Color online) Energy-level diagram of PFP/Ag(111) from UPS. The vacuum level $\left(E_{v a c}\right)$, the Fermi level $\left(E_{F}\right)$, vacuumlevel shifts $\left(\Delta_{v a c}\right)$ and HOMO positions are plotted as function of the nominal film thickness. For the pristine $\operatorname{Ag}(111)$ substrate the work function is indicated, for the PFP film the ionization energy is given (both in $\mathrm{eV}$ ). As the multilayer spectra are superpositions of spectral features from different thickness regions, the HOMO onset was estimated by the peak maximum plus $\sim 0.15 \mathrm{eV}$. The values in red correspond to the IE of "flat lying" (i.e., the IE of the second flat lying layer, without contributions from substrate photohole screening) and "herringbone" (i.e., the IE of multilayer PFP without contributions from flat lying layers) of PFP on $\operatorname{Ag}(111)$.

position of spectral features from different layers in the UPS spectra for multilayer coverage. For all coverages the intensity of the HOMO and HOMO-1 derived peaks was rather weak for normal emission and significantly increased for $\theta$ $=45^{\circ}$. This behavior is commonly observed for flat lying molecules. ${ }^{12,38,39}$ However, for coverages $>6 \AA$ the relative intensity in the normal-emission spectra increased, which can be attributed to molecules with a tilt of their $\pi$-system with respect to the substrate surface. This supports a herringbone arrangement of PFP (as concluded from XRD) that prevails for the nominally third molecular layer onwards.

\section{DISCUSSION}

The structure scenario as deduced from XSW and XRD can be summarized as follows: The monolayer PFP/Ag(111) is lying flat, whereas multilayers adopt a molecular orientation with the short molecular axis tilted, most likely in the herringbone arrangement. A sudden orientational transition of rodlike molecules on metal surfaces with increasing film thickness is frequently observed after the monolayer, ${ }^{12,14,19}$ however, the critical coverage for this transition is difficult to predict. ${ }^{14,40}$ However, as discussed in Sec. III, it is reasonable that the IE (Ref. 59) change from 5.80 to $6.10 \mathrm{eV}$ [Fig. $4]$ is not due to an orientational transition but only due to decreased photohole screening, and the herringbone arrangement of PFP/Ag(111) starts with the third molecular layer.

As expected, the molecular plane tilt in multilayers leads to an increase of the IE of PFP [Fig. 4] due to the strong IPB character of the carbon-fluorine bonds. The increase in the IE even leads to a change in the energy-level alignment from 
vacuum-level controlled (lying PFP) to Fermi-level pinned $^{41-43}$ (herringbone PFP) as was inferred from the 0.12 $\mathrm{eV}$ vacuum-level shift between bilayer and multilayer coverage. A similar spontaneous charge transfer at an organic/ organic homointerface due to an orientational transition has been observed before, ${ }^{44}$ however with opposite direction. For PFP in the herringbone arrangement the small transport gap (estimated to be $\sim 2.10 \mathrm{eV}$ ) (Ref. 10) and the large IE pins the PFP lowest unoccupied molecular orbital (LUMO) just above the Fermi level (i.e, without the $0.12 \mathrm{eV}$ vacuum-level shift, for the $50 \AA$ film the LUMO would be below the Fermi level).

The increase in IE from the flat lying to the herringbone structure observed here is only $0.45 \mathrm{eV}$ compared to $0.65 \mathrm{eV}$ IE difference between lying and nearly upright standing molecular layers. ${ }^{7,12}$ This can be explained by the different inclination angles of the IPBs of the two systems. In a recent theoretical study on the IE of fluorinated poly(3hexylthiophene) the IE increased with increasing inclination angles and the dependency could be well reproduced by a simple electrostatic model. ${ }^{45} \mathrm{~A}$ similar behavior can be expected for the small molecule PFP and was observed for other molecules before: For 3,4,9,10-perylene tetracarboxyilic dianhydride the IE changes by $0.15 \mathrm{eV}$ by a change in the inclination angle to a substrate by just $\sim 10^{\circ}$ (Ref. 46). Even for the only slightly different thin-film polymorphs and the bulk phase of the unfluorinated parent molecule pentacene different IEs were measured. ${ }^{47}$ Hence, it can be assumed that the impact of IPBs of rodlike molecules on the electronic structure of an organic thin film depends on the inclination angle relative to a substrate. If the IPBs along the molecule's long and short axes have the same character [as it is the case for PFP, see Fig. 1(a)], the IE is affected by the IPBs in a similar way, irrespective of whether the molecules are inclined with their short or long molecular axis.

Beyond the impact of PEN fluorination on the electronic structure via the strong IPB character of the carbon-fluorine bond, fluorination also impacts the adsorbate-substrate interaction. The valence electron spectra of PFP on $\mathrm{Au}(111),{ }^{12}$ $\mathrm{Ag}(111)$, and $\mathrm{Cu}(111)$ (Ref. 21) are very similar, irrespective of the wide range of substrate work function and chemical reactivity of these metal substrates, whereas the UPS spectra of PEN/Ag(111) (Ref. 48) are in strong contrast to PFP/ $\mathrm{Ag}(111)$. The IPB character of the carbon-hydrogen bond is much smaller than that of carbon-fluorine and has an opposite sign. ${ }^{7,10}$ Therefore, the IE of PEN is smaller for standing molecules compared to lying molecules., ${ }^{78,49}$ Furthermore, for various single crystalline metal substrates the distinct physical properties of the substrates are directly reflected in the PEN thin-film spectra. ${ }^{12,21,38,50-52}$ The bonding distances are significantly smaller for PEN than for PFP on $\mathrm{Ag}(111)$ (Ref. 53) as on $\mathrm{Cu}(111){ }^{21}$ The bonding distances of PFP/ $\mathrm{Cu}(111)$ are $2.98 \AA$ for carbon and $3.08 \AA$ for fluorine. ${ }^{21} \mathrm{In}$ this case the smaller bonding distance [compared to PFP/ $\mathrm{Ag}(111)]$ leads to a molecular distortion even for a relatively weakly interacting system. This shows the dramatic impact of fluorination on the reactivity of PEN: The PFP-substrate interaction is weak on all metals and close to physisorption, whereas the PEN-substrate interaction is stronger in all cases and-at least - in contact to $\mathrm{Cu}$ substrates exhibits clear evidence for chemisorption.

\section{CONCLUSIONS}

Our multitechnique study revealed a flat lying orientation of the PFP monolayer on $\mathrm{Ag}(111)$ with an average bonding distance of $3.16 \AA$. Multilayers adopt a herringbonelike arrangement, which leads to an increase in the PFP ionization energy. Thus, it is demonstrated that the concept of intramolecular polar bonds is also valid for molecular arrangements in a herringbone structure with their molecular long axis oriented parallel to the substrate surface, and accordingly impacts the ionization energy. Therewith, our results highlight the importance of knowing the exact molecular orientation at organic/metal interfaces when discussing interface energetics since the ionization energy is not an intrinsic material property and cannot be treated delineated from molecular layer structure.

\section{ACKNOWLEDGMENTS}

The authors thank Wolfgang Caliebe (HASYLAB) for assistance during XRD measurements. S.D. gratefully acknowledges a JSPS fellowship for foreign researchers. N.K. acknowledges financial support by the Emmy NoetherProgram (DFG), S.K. by a Grant-in-Aid for Young Scientists (A), B.W. by the Austrian Science Foundation (FWF) (Project No. P21094), F.S. and A.G. by the DFG. This work was financially supported partly by the Global-COE Program of MEXT (Advanced School for Organic Electronics, Chiba University) and the DFG (Sfb448 and SPP1355).

\footnotetext{
*duhm@ restaff.chiba-u.jp

${ }^{\dagger}$ Present address: Diamond Light Source Ltd, Harwell Science and Innovation Campus, Oxfordshire, OX11 0DE, UK.

${ }^{1}$ J. Hwang, A. Wan, and A. Kahn, Mater. Sci. Eng., R. 64, 1 (2009).

${ }^{2}$ H. Ishii, K. Sugiyama, E. Ito, and K. Seki, Adv. Mater. 11, 605 (1999).

${ }^{3}$ N. Koch, ChemPhysChem 8, 1438 (2007).

${ }^{4}$ X. Crispin, V. Geskin, A. Crispin, J. Cornil, R. Lazzaroni, W. R. Salaneck, and J.-L. Brédas, J. Am. Chem. Soc. 124, 8131 (2002).
}

${ }^{5}$ A. Kahn, N. Koch, and W. Y. Gao, J. Polym. Sci., Part B: Polym. Phys. 41, 2529 (2003).

${ }^{6}$ N. Ueno and S. Kera, Prog. Surf. Sci. 83, 490 (2008).

${ }^{7}$ I. Salzmann, S. Duhm, G. Heimel, M. Oehzelt, R. Kniprath, R. L. Johnson, J. P. Rabe, and N. Koch, J. Am. Chem. Soc. 130, 12870 (2008).

${ }^{8}$ S. Duhm, G. Heimel, I. Salzmann, H. Glowatzki, R. L. Johnson, A. Vollmer, J. P. Rabe, and N. Koch, Nature Mater. 7, 326 (2008).

${ }^{9}$ W. Chen, H. Huang, S. Chen, Y. L. Huang, X. Y. Gao, and A. T. S. Wee, Chem. Mater. 20, 7017 (2008). 
${ }^{10}$ S. Duhm, I. Salzmann, G. Heimel, M. Oehzelt, A. Haase, R. L. Johnson, J. P. Rabe, and N. Koch, Appl. Phys. Lett. 94, 033304 (2009).

${ }^{11}$ Y. Sakamoto, T. Suzuki, M. Kobayashi, Y. Gao, Y. Fukai, Y. Inoue, F. Sato, and S. Tokito, J. Am. Chem. Soc. 126, 8138 (2004).

${ }^{12}$ N. Koch, A. Vollmer, S. Duhm, Y. Sakamoto, and T. Suzuki, Adv. Mater. 19, 112 (2007).

${ }^{13}$ F. Schreiber, Phys. Status Solidi A 201, 1037 (2004).

${ }^{14}$ G. Witte and C. Wöll, J. Mater. Res. 19, 1889 (2004).

${ }^{15}$ D. E. Hooks, T. Fritz, and M. D. Ward, Adv. Mater. 13, 227 (2001).

${ }^{16}$ G. Beernink, T. Strunskus, G. Witte, and C. Wöll, Appl. Phys. Lett. 85, 398 (2004).

${ }^{17}$ C. E. Heiner, J. Dreyer, I. V. Hertel, N. Koch, H.-H. Ritze, W. Widdra, and B. Winter, Appl. Phys. Lett. 87, 093501 (2005).

${ }^{18}$ L. Casalis, M. F. Danisman, B. Nickel, G. Bracco, T. Toccoli, S. Iannotta, and G. Scoles, Phys. Rev. Lett. 90, 206101 (2003).

${ }^{19}$ S. Duhm, H. Glowatzki, J. P. Rabe, N. Koch, and R. L. Johnson, Appl. Phys. Lett. 88, 203109 (2006).

${ }^{20}$ J.-Z. Wang, J. T. Sadowski, Z.-H. Xiong, Y. Fujikawa, Q. K. Xue, and T. Sakurai, Nanotechnology 20, 095704 (2009).

${ }^{21}$ N. Koch et al., J. Am. Chem. Soc. 130, 7300 (2008).

${ }^{22}$ D. P. Woodruff, Rep. Prog. Phys. 68, 743 (2005).

${ }^{23}$ J. Zegenhagen, Surf. Sci. Rep. 18, 202 (1993).

${ }^{24}$ C. Stadler, S. Hansen, I. Kröger, C. Kumpf, and E. Umbach, Nat. Phys. 5, 153 (2009).

${ }^{25}$ A. Hauschild, K. Karki, B. C. C. Cowie, M. Rohlfing, F. S. Tautz, and M. Sokolowski, Phys. Rev. Lett. 94, 036106 (2005).

${ }^{26}$ A. Gerlach, S. Sellner, F. Schreiber, N. Koch, and J. Zegenhagen, Phys. Rev. B 75, 045401 (2007).

${ }^{27}$ A. Gerlach, F. Schreiber, S. Sellner, H. Dosch, I. A. Vartanyants, B. C. C. Cowie, T.-L. Lee, and J. Zegenhagen, Phys. Rev. B 71, 205425 (2005).

${ }^{28}$ S. Schiefer, M. Huth, A. Dobrinevski, and B. Nickel, J. Am. Chem. Soc. 129, 10316 (2007).

${ }^{29}$ S. Kowarik, A. Gerlach, and F. Schreiber, J. Phys.: Condens. Matter 20, 184005 (2008).

${ }^{30}$ I. Salzmann, S. Duhm, G. Heimel, J. P. Rabe, N. Koch, M. Oehzelt, Y. Sakamoto, and T. Suzuki, Langmuir 24, 7294 (2008).

${ }^{31}$ S. Kowarik, A. Gerlach, A. Hinderhofer, S. Milita, F. Borgatti, F. Zontone, T. Suzuki, F. Biscarini, and F. Schreiber,Phys. Status Solidi RRL 2, 120 (2008).

${ }^{32}$ P. S. Bagus, V. Staemmler, and C. Wöll, Phys. Rev. Lett. 89, 096104 (2002).

${ }^{33}$ S. Kera, H. Yamane, and N. Ueno, Prog. Surf. Sci. 84, 135 (2009).

${ }^{34}$ K. Fujii, C. Himcinschi, M. Toader, S. Kera, D. R. T. Zahn, and N. Ueno, J. Electron Spectrosc. Relat. Phenom. 174, 65 (2009).

${ }^{35}$ N. Koch, G. Heimel, J. Wu, E. Zojer, R. L. Johnson, J.-L. Brédas, K. Müllen, and J. P. Rabe, Chem. Phys. Lett. 413, 390 (2005).

${ }^{36}$ I. G. Hill, A. J. Mäkinen, and Z. H. Kafafi, J. Appl. Phys. 88,
889 (2000)

${ }^{37}$ N. Koch, J. Phys.: Condens. Matter 20, 184008 (2008).

${ }^{38}$ O. McDonald, A. A. Cafolla, Z. Li, and G. Hughes, Surf. Sci. 600, 1909 (2006).

${ }^{39}$ S. Kera, H. Yamane, H. Fukagawa, T. Hanatani, K. Okudaira, K. Seki, and N. Ueno, J. Electron Spectrosc. Relat. Phenom. 156158, 135 (2007).

${ }^{40}$ S. Duhm, I. Salzmann, N. Koch, H. Fukagawa, T. Kataoka, S. Hosoumi, K. Nebashi, S. Kera, and N. Ueno, J. Appl. Phys. 104, 033717 (2008).

${ }^{41}$ N. Koch and A. Vollmer, Appl. Phys. Lett. 89, 162107 (2006).

${ }^{42}$ H. Fukagawa, S. Kera, T. Kataoka, S. Hosoumi, Y. Watanabe, K. Kudo, and N. Ueno, Adv. Mater. 19, 665 (2007).

${ }^{43}$ S. Braun, W. R. Salaneck, and M. Fahlman, Adv. Mater. 21, 1450 (2009).

${ }^{44}$ S. Duhm, H. Glowatzki, J. P. Rabe, N. Koch, and R. L. Johnson, Appl. Phys. Lett. 90, 122113 (2007).

${ }^{45}$ G. Heimel, I. Salzmann, S. Duhm, J. P. Rabe, and N. Koch, Adv. Funct. Mater. 19, 3874 (2009).

${ }^{46}$ W. Chen, D. C. Qi, Y. L. Huang, H. Huang, Y. Z. Wang, S. Chen, X. Y. Gao, and A. T. S. Wee, J. Phys. Chem. C 113, 12832 (2009).

${ }^{47}$ H. Yoshida and N. Sato, Phys. Rev. B 77, 235205 (2008).

${ }^{48}$ N. Koch, I. Salzmann, R. L. Johnson, J. Pflaum, R. Friedlein, and J. P. Rabe, Org. Electron. 7, 537 (2006).

${ }^{49}$ H. Fukagawa, H. Yamane, T. Kataoka, S. Kera, M. Nakamura, K. Kudo, and N. Ueno, Phys. Rev. B 73, 245310 (2006).

${ }^{50}$ H. Yamane, D. Yoshimura, E. Kawabe, R. Sumii, K. Kanai, Y. Ouchi, N. Ueno, and K. Seki, Phys. Rev. B 76, 165436 (2007).

${ }^{51}$ M. G. Betti, A. Kanjilal, and C. Mariani, J. Phys. Chem. A 111, 12454 (2007).

${ }^{52}$ H. Yamane, K. Kanai, Y. Ouchi, N. Ueno, and K. Seki, J. Electron Spectrosc. Relat. Phenom. 174, 28 (2009).

${ }^{53}$ S. Duhm, A. Gerlach, N. Koch, and F. Schreiber (unpublished).

${ }^{54}$ P. Trucano and R. Chen, Nature (London) 258, 136 (1975).

${ }^{55}$ S. Duhm, I. Salzmann, R. L. Johnson, and N. Koch, J. Electron Spectrosc. Relat. Phenom. 174, 40 (2009).

${ }^{56}$ G. Koller, B. Winter, M. Oehzelt, J. Ivanco, F. P. Netzer, and M. G. Ramsey, Org. Electron. 8, 63 (2007).

${ }^{57}$ R. Fischer, S. Schuppler, N. Fischer, T. Fauster, and W. Steinmann, Phys. Rev. Lett. 70, 654 (1993).

${ }^{58}$ Note that the (002)-interlayer distance of graphite $(\mathrm{d}=3.56 \AA)$ (Ref. 54), which can be denoted as the prototypical stacked organic molecular crystal, is even slightly larger than the sum of the van der Waals radii.

${ }^{59}$ Note that in complex structures (here: island growth plus orientational transition) the exact correlation of the SECO position to a certain organic layer may be difficult (Ref. 55) since in the valence electron region the UPS spectra are a superposition of emission from sample patches with different spectral feature BEs, whereas the vacuum-level position is an area averaged arithmetic mean of sample patches with different vacuum levels (Refs. 56 and 57). Thus the ionization energy values in Fig. 4 should be dealt with carefully. 